Objectives

1. To investigate the documentation of code status discussion on admission to hospital.

2. To investigate patient outcomes in those referred to Palliative care services.

Methods The electronic health records (EHR) of 50 patients referred to the Palliative Care consult service were retrospectively reviewed, following Institutional Review Board (IRB) approval. The patients code status orders, clinical notes, and outcomes were documented.

Results The average age of the patients was 67.86 years. $48 \%$ had an active cancer diagnosis, $10 \%$ were left ventricular device (LVAD) patients and $42 \%$ had an acute cardiac, pulmonary or neurological presentation. $34 \%$ of patients were admitted directly to an intensive care setting. The majority $(80 \%)$ of patients were 'Full Code' on admission, 12\% were 'Do Not Resuscitate' (DNR) and 8\% were 'Partial Code'. For those who were documented as 'DNR', only 1 patient had a documented 'Do Not Intubate' (DNI) order. While code status was documented in all admission notes, the discussion of code status clarification was only documented in $14 \%$ of notes. Only one emergency department note referenced a discussion of code status. Code status was changed within 24 hours in $6 \%$ of patients, and $20 \%$ of patients changed code status during admission, with Palliative Care involvement in the majority $(80 \%)$ of these cases. Regarding outcomes, 56\% returned home, 14\% were discharged for rehabilitation, $22 \%$ were discharged with hospice care, and $6 \%$ died in hospital on comfort care.

Conclusion Code status discussion should be an integral part of an acute hospital admission to ensure patient's wishes are being followed and respected.

\section{COMPLIANCE WITH HOSPITAL GUIDELINES ON PRESCRIPTION OF ANTICIPATORY MEDICATIONS - SCOPE FOR IMPROVEMENT?}

Manisha Patel, Sally Sutcliffe, Mariam George. Tameside and Glossop Integrated Care Foundation Trust

\subsection{6/bmjspcare-2019-ASP.84}

Background NICE guidelines for the Care of the Dying Adult recommend the prescription of anticipatory medication as early as possible to minimise symptomatic distress during last days of life. Literature on prescribing of anticipatory medications in secondary care is limited. Incorrect prescribing and inadequate understanding among hospital staff regarding symptom management for a dying patient prompted the need to establish compliance of prescribing and administration of anticipatory medications (AMs) against Trust guidelines on symptom control in the last days of life.

Method A retrospective audit cycle comprising of 3 audits was conducted examining hospital records of patients recognized as in the dying phase, between December 2015 and October 2017. Documentation within drug charts, doctors' and nursing notes were scrutinised to establish whether AMs were prescribed and used for specific indications as per guidelines.

Results There was improvement in compliance with prescribing of AMs for patients at point of recognition of being in the dying phase $(88 \%$ to $96 \%)$ and the administration of AMs according to correct indications (23\% to 91\%). Nursing documentation of indication for AM use improved (23\% to 40\%). Despite an improvement in selection of appropriate AM per symptom, compliance around doctors' prescription of accurate doses dropped (21\% to $12 \%)$.

Conclusions Whilst compliance in the initiation of anticipatory medications for the main symptoms associated with last days of life improved, accuracy of doses needs to improve. The role of hospital pharmacists in enabling compliance was identified as an additional resource alongside periodic trust-wide communication of guidelines, to support better symptom control at end of life. The prevalence of hospital staff turnover (doctors changing rotations and bank nurses) necessitates the need for regular educational events to facilitate safe, evidence based symptom control at end of life. Embedding clinical guidelines for end of life care requires an ongoing proactive approach.

\section{2 'PLEASE, I WANT TO GO HOME' - CREATING A HOMECARE TEAM TO EXPEDITE DISCHARGE HOME FROM HOSPITAL AT THE END OF LIFE}

Magdalen Thomson, Sarah McGaughey, Tracey Platt, Rachel Gaffney. Hampshire Hospitals NHS Foundation Trust

\subsection{6/bmjspcare-2019-ASP.85}

Background The project addressed delays in discharge and its impact on unnecessary acute bed occupancy, responding to 'Please, I want to go home' for dying patients. The press perceptions that the 'NHS must get a grip on hospital discharge delays', and discharge delay rise by $23 \%$ in the preceding year (NHS England) led to agreement that it was unacceptable that vulnerable and dying patients were 'stuck' in hospital.

Method A case for change put to our local CHC commissioners highlighted the lack of care capacity, CCG spot purchasing methodology, and patients with agreed fast track (FT) funding being inappropriately admitted into hospital as no care available at home. FT applications were increasing yearly, the CHC department was understaffed and challenged to meet the NHS CHC Framework timeframes, and the FT reviews were not being undertaken within 3 months. Some individuals had been on the FT pathway for 3 years. Commissioner contract aims were agreed assuring quality of service provision, a responsive service to meet individual needs, implementation within 48 hours, and over seen by the hospital palliative care team. Continuity of care, review of FT eligibility and dynamic care delivery, assured best value of NHS CHC resources whilst improved patient experience.

Results In March 2016 The Homecare service was set up within Winchester and Andover, funded by $\mathrm{CHC}$ fast track. The first patient was accepted on 18th July 2016.

In the first 6 months the service supported 32 patients, delivered 1,147 hours of care by Band 3 healthcare support workers 8 am to $8.30 \mathrm{pm}$, and supported by the hospital/hospice palliative care team. Fast track discharge delays were reduced from 15.3 days to less than 2 days.

Conclusion This service enabled swift patient discharge, met preference, and provided timely, high quality, flexible care. It also improved patient flow through Andover and Winchester hospitals. 